\title{
ARrico $\mid$ Do pré-natal ao parto: um estudo transversal sobre a influência do acompanhante nas boas práticas obstétricas no Sistema Único de Saúde em Santa Catarina, 2019*
}

From prenatal care to childbirth: a cross-sectional study on the influence of a companion on good obstetric practices in the Brazilian National Health System in Santa Catarina State, 2019

Desde la atención prenatal hasta el parto: un estudio transversal sobre la influencia del acompañante en las buenas prácticas obstétricas el Sistema Único de Salud en Santa Catarina, Brasil, 2019

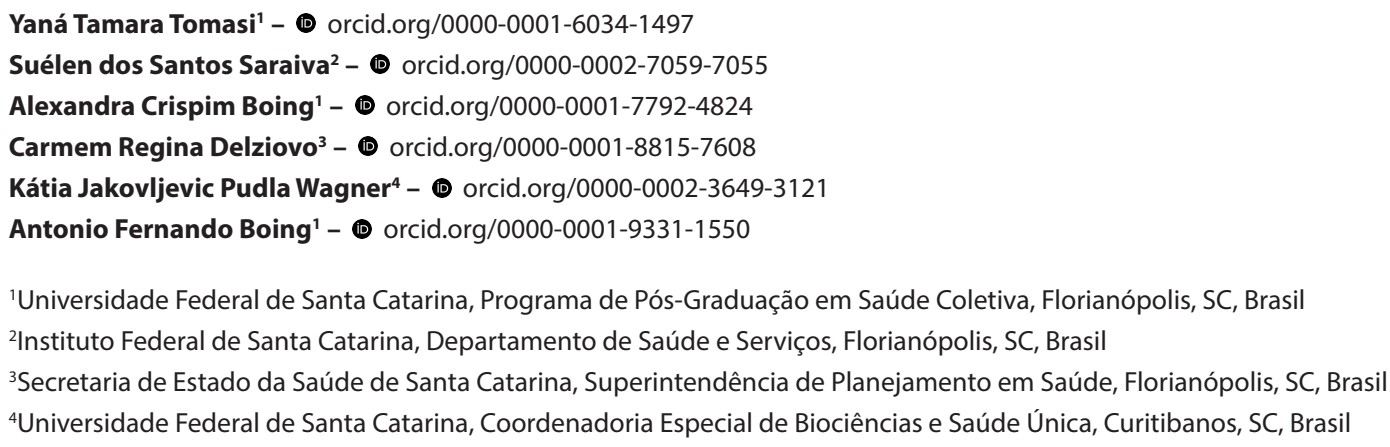

\section{Resumo}

Objetivo: Analisar a associação da presença de acompanhante no pré-natal e parto com a qualidade da assistência recebida por usuárias do Sistema Único de Saúde (SUS). Métodos: Estudo transversal com puérperas que realizaram pré-natal e parto pelo SUS em Santa Catarina, Brasil, em 2019, entrevistadas em até 48 horas após o parto. Estimaram-se as razões de prevalências mediante regressão de Poisson. Resultados: Entrevistaram-se 3.580 puérperas. No pré-natal, a presença de acompanhante associou-se positivamente ao recebimento de orientações pelos profissionais da saúde $\left(\mathrm{RP}=1,27-\mathrm{IC}_{95 \%}\right.$ $1,08 ; 1,50)$ e à construção do plano de parto $\left(\mathrm{RP}=1,51-\mathrm{IC}_{05 \%}, 1,15 ; 1,97\right)$. No parto, a presença de acompanhante associou-se ao maior recebimento de analgesia $\left(\mathrm{RP}=2,89-\mathrm{IC}_{95 \%}, 1,40 ; 5,97\right)$, manobra não farmacológica para alívio da dor $(\mathrm{RP}=1,96$ - $\left.\mathrm{IC}_{95 \%} 1,44 ; 2,65\right)$, escolha da posição para o parto $\left(\mathrm{RP}=1,63-\mathrm{IC}_{95 \%}, 1,24 ; 2,16\right)$ e menor probabilidade de ser amarrada $\left(\mathrm{RP}=0,47-\mathrm{IC}_{95 \%}, 0,35 ; 0,63\right)$. Conclusão: A presença de acompanhante no pré-natal e parto mostrou-se associada à melhor qualidade da assistência.

Palavras-chave: Cuidado Pré-Natal; Parto Humanizado; Sistema Único de Saúde; Direitos do Paciente; Saúde da Mulher; Estudos Transversais.

*Estudo financiando pela Fundação de Amparo à Pesquisa de Santa Catarina:Termo de Outorga n² 2017 TR1364.

\section{Endereço para correspondência:}

Yaná Tamara Tomasi - Universidade Federal de Santa Catarina, Programa de Pós-Graduação em Saúde Coletiva, Campus Reitor João David Ferreira Lima, Trindade, Florianópolis, SC, Brasil. CEP: 88040-900

E-mail: yanaatomasi@gmail.com 


\section{Introdução}

A oferta de assistência à saúde com qualidade e humanizada, durante o ciclo gravídico puerperal, é essencial se se pretende obter bons resultados clínicos para a mãe e 0 recém-nascido. Ela está associada à menor morbimortalidade e à não ocorrência de intervenções médicas desnecessárias, além de ter efeitos positivos no trabalho de parto e na sensação e sentimento de controle pela mulher. ${ }^{1,2}$

\section{A presença do acompanhante durante 0 período gravídico-puerperal transmite à mulher mais segurança, além de contribuir para melhores desfechos maternos e neonatais.}

Uma das ações que visam contribuir para a melhoria da assistência em saúde no período de gravidez e no parto, e tem sido objeto de reforço legal e infralegal, por meio de normativas e orientações, é a presença de acompanhante da mulher, nas condições de gestante e parturiente, nos serviços de saúde. A presença do acompanhante durante o período gravídico-puerperal transmite à mulher mais segurança, além de contribuir para melhores desfechos maternos e neonatais. ${ }^{2}$

A Organização Mundial da Saúde (OMS) recomenda a presença de acompanhante de livre escolha da gestante como uma das ações de boas práticas de atenção ao parto e nascimento, com vistas à redução de intervenções desnecessárias e violências obstétricas. ${ }^{3}$

Considerando-se essa recomendação e sua importância, a presença de acompanhante de escolha da mulher, durante o período de trabalho de parto, parto e pós-parto imediato, foi regulamentada no Brasil, por meio da Lei $\mathrm{n}^{0} 11.108$, de 7 de abril de 2005. Esta medida é reafirmada pela Portaria GM/MS $\mathrm{n}^{0} 1.459$, de 24 de junho de 2011, que institui a Rede Cegonha no âmbito do Sistema Único de Saúde (SUS) com 0 propósito de garantir parto e nascimento seguros às mulheres e seus recém-nascidos, a partir da presença de acompanhante de sua livre escolha., 4

Para além do acompanhamento no momento do parto, a Rede Cegonha prevê, como estratégias fundamentais para qualificar o cuidado à mulher e ao feto, orientações sobre 0 direito a acompanhante durante todo o ciclo gravídico-puerperal - o que inclui o período pré-natal. ${ }^{2}$ Consultas de pré-natal com a presença de acompanhante representam um momento de fortalecimento de vínculos entre equipe de saúde, gestante e acompanhante, além de representarem uma oportunidade de capacitação para 0 parto. Adicionalmente, tem-se verificado que contar com a presença de acompanhante durante o pré-natal é fator preditivo para a gestante também estar acompanhada durante o parto, indicando a importância de se estimular e viabilizar o acompanhamento desde 0 início do cuidado e não apenas no momento do nascimento da criança. ${ }^{6}$

Apesar de o Brasil apresentar aumento na proporção de gestantes com pelo menos sete consultas de pré-natal e parto assistido por profissionais de saúde, ainda há necessidade de aprimorar a qualidade dessas consultas e o cuidado no parto e pós-parto. ${ }^{78}$ A presença de acompanhantes no ciclo gravídico-puerperal pode, além de ser uma intervenção segura e de baixo custo, melhorar a qualidade do cuidado oferecido à mulher e à criança. ${ }^{6,9}$

São escassos os estudos que analisam a associação entre acompanhamento no pré-natal e as práticas assistenciais desenvolvidas. ${ }^{10}$ Predominam investigações qualitativas ou voltadas à experiência vivenciada e sua avaliação por acompanhante ou pelos serviços de saúde. ${ }^{11-13}$ Pesquisas quantitativas, com amostra representativa, não estão disponíveis. Estudos populacionais, ao analisarem a associação da presença de acompanhante no parto e pós-parto com desfechos obstétricos, demonstraram uma relação positiva entre esse acompanhamento, boas práticas assistenciais e desfechos clínicos satisfatórios, embora sejam igualmente escassos. ${ }^{2,9}$

0 presente estudo teve como objetivo analisar a associação da presença de acompanhante, durante as consultas de pré-natal e no parto, com a qualidade da assistência recebida por usuárias do SUS.

\section{Métodos}

Foi realizado um estudo transversal, cuja população observada foi de puérperas com filhos nascidos em hospitais do estado de Santa Catarina no ano de 2019, independentemente da idade. Santa Catarina localiza-se na região Sul do país e conta com uma população estimada de 7.164 .788 habitantes em 2019, 
distribuídos entre 295 municípios (https:/www.ibge. gov.br/cidades-e-estados/sc.html). No mesmo ano de 2019, nasceram vivas 97.589 crianças no estado, $57,20 \%$ delas pelo procedimento cirúrgico de cesariana (http://200.19.223.105/cgi-bin/tabnet?sinasc/def/ sinasc.def).

Os critérios para inclusão no estudo foram: durante todo o período gestacional, ter mantido residência em Santa Catarina; realização de todas as consultas de pré-natal (seja gravidez de risco habitual, seja gravidez de alto risco) na rede pública de saúde; realização do parto em hospital do estado que teve mais de 500 partos via SUS, no ano de 2016; e filho nascido vivo, natimorto ou morto até 48 horas após o parto, com mais de $500 \mathrm{~g}$ e pelo menos 22 semanas de gestação.

Por conseguinte, foram incluídos no estudo todos os 31 hospitais de Santa Catarina que realizaram 500 ou mais partos pelo SUS em 2016, distribuídos em 30 diferentes municípios. No conjunto, esses hospitais concentraram $86,2 \%$ de todos os nascimentos no estado com financiamento do SUS, naquele ano. Assim, estimou-se como amostra total do estudo 3.665 puérperas. Considerou-se um intervalo de confiança de $95 \%$, margem de erro de 1,6 ponto percentual, tamanho populacional de 50 mil hab. e prevalência esperada do fenômeno de 50\%, para majoração da amostra final. Assim estimada, a amostra ainda foi acrescida em 5\% para compensar eventuais perdas e recusas. A definição do quantitativo de entrevistas realizadas em cada hospital seguiu a distribuição proporcional de nascimentos observada em 2016.

Para a coleta de dados, questionários fechados foram aplicados face a face com as puérperas, nos hospitais, até 48 horas pós-parto, por meio de tablets e com registro na plataforma Research Eletronic Data Capture (REDCap). A logística do campo foi testada em estudo-piloto com $5 \%$ da amostra. No total, 35 entrevistadores, todos com nível superior completo, ou em curso, e experiência na área da Saúde, foram treinados e participaram da coleta dos dados. Para controle de qualidade, entrevistou-se amostra aleatória de 10\% das participantes, por telefone. Ao se analisar as variáveis do controle de qualidade, observou-se boa ou quase perfeita concordância: entre as oito variáveis analisadas, seis apresentaram coeficiente de concordância de Kappa de Cohen superior a 0,680.

As variáveis de desfecho foram relacionadas a dois momentos do período gravídico: o pré-natal e o parto.
Sobre o pré-natal, analisou-se a construção do plano de parto com a(o) profissional de saúde e o recebimento, pela gestante, de ao menos uma das orientações recomendadas pelo Ministério da Saúde, em seu Caderno de Atenção Básica $n^{0} 32$, sobre Atenção ao Pré-Natal de Baixo Risco, quais sejam: (i) a importância da amamentação exclusiva até os 6 meses de idade do bebê, (ii) a forma de posicionar o bebê para amamentar, (iii) a importância da prática de atividades físicas durante a gestação, (iv) os riscos da utilização de medicamentos durante a gestação, (v) os riscos do consumo de álcool durante esse período, (vi) os riscos do consumo de tabaco durante esse período, (vii) os sinais de risco para os quais se deve procurar por assistência, (viii) os sinais de início do trabalho de parto, (ix) 0 que se pode fazer durante o trabalho de parto para facilitar 0 nascimento, (x) a possibilidade da presença de acompanhante durante o período do parto e no pós-parto e (xi) a possibilidade de visitar a maternidade durante 0 pré-natal. ${ }^{14}$

Quanto ao parto, foram analisados, como desfechos, (i) a amamentação durante a primeira hora de vida do bebê, (ii) 0 ato de ser amarrada durante 0 parto; e, para os casos de via de parto vaginal, (iii) a realização de episiotomia, (iv) a possibilidade de escolher a posição de expulsão, (v) o recebimento de manobra não farmacológica para alívio da dor (chuveiro, bola e massagem) e (vi) a analgesia.

Todas as informações foram dicotômicas (sim ou não), autorreferidas pela mulher.

A variável explicativa de principal interesse foi a presença de acompanhante nas consultas de pré-natal, seja na maioria, seja em algumas dessas consultas (sim ou não), e durante o parto (sim ou não). As variáveis de ajuste foram a faixa etária (em anos: 13-19; 20-35; 36-46), morar com companheiro (sim ou não), planejamento da gravidez (sim ou não), raça/cor da pele autorreferida (branca, preta ou parda; indígenas e amarelas não foram analisadas, pela baixa quantidade de entrevistas), anos de estudo ( $\geq 13$; $10-12 ; \leq 9$ ), renda per capita (em reais [R\$], categorizada em tercis) e número de consultas de pré-natal (1-3; 4-6; 7 ou mais).

$\mathrm{Na}$ análise estatística, inicialmente foram calculadas a distribuição da amostra e as prevalências dos desfechos segundo a presença ou não de acompanhante no pré-natal e no parto, com seus respectivos intervalos de confiança de $95 \%\left(\mathrm{IC}_{95 \%}\right)$. 
Em seguida foi calculada a razão de prevalências (RP) da presença de acompanhante no pré-natal e no parto, por meio da regressão de Poisson, nos modelos bruto e ajustado, sendo as medidas reportadas com seus respectivos $\mathrm{IC}_{95 \%}$. Todas as análises foram realizadas com o uso do software Stata 15.1.

0 projeto da pesquisa recebeu a aprovação do Comitê de Ética em Pesquisa com Seres Humanos da Universidade Federal de Santa Catarina (CEPSH/UFSC) em 20 de junho de 2016 - Parecer ${ }^{0} 1.599 .464$-, e seguiu integralmente os preceitos éticos da Resolução do Conselho Nacional de Saúde (CNS) no 466, de 12 de dezembro de 2012. As puérperas que aceitaram participar da pesquisa assinaram um Termo de Consentimento Livre e Esclarecido (TCLE) digitalmente, utilizando o tablet do pesquisador, e receberam cópia impressa de seu consentimento no momento da entrevista.

\section{Resultados}

Foram entrevistadas 3.580 puérperas, após 85 recusas (taxa de resposta: 97,7\%). Observou-se que 13,4\% delas eram adolescentes, duas em cada três se autorreferiram brancas, quatro em cada cinco moravam com companheiro, $40 \%$ haviam planejado a gravidez e $65,5 \%$ tinham concluído o ensino fundamental (Tabela 1). Em 57,2\% dos nascimentos, o tipo de parto foi vaginal, e quatro em cada cinco mulheres realizaram sete ou mais consultas de pré-natal.

Apenas $17,5 \%$ das puérperas receberam dos profissionais de saúde todas as orientações previstas para 0 acompanhamento pré-natal (Tabela 2). As orientações menos frequentes foram sobre a possibilidade de a mulher visitar a maternidade durante 0 pré-natal (40,3\%), como posicionar o bebê para mamar (46,6\%) e 0 que se pode fazer durante 0 trabalho de parto para facilitar 0 nascimento $(52,0 \%)$. Entre as orientações mais comuns, destacaram-se aquelas sobre os sinais de risco durante a gravidez que devem fazer a gestante procurar por assistência $(80,5 \%)$, os riscos de utilizar medicamentos $(77,2 \%)$, fumar $(75,6 \%)$ e consumir álcool $(75,0 \%)$ durante a gravidez. Apenas uma em cada 13 puérperas referiu a elaboração de plano de parto com a $(0)$ profissional de saúde durante 0 pré-natal. Em todos os desfechos, houve maior prevalência de orientações para as mulheres quando acompanhadas nas consultas.
Em relação ao parto, verificou-se que $71,2 \%$ das puérperas amamentaram na primeira hora após 0 nascimento do bebê e nove em cada 100 foram amarradas durante o parto (Tabela 3). Ao se analisar as mulheres que realizaram parto vaginal, a episiotomia foi realizada em $14,3 \%$ delas e $49,0 \%$ puderam escolher sua posição de parto. Apenas em $18,4 \%$ das parturientes, aplicou-se analgesia, e em 52,7\% delas decidiu-se por alguma medida não farmacológica para alívio da dor, sendo o acesso ao chuveiro a mais comum (48,7\%). Todos os desfechos positivos para a mulher e 0 bebê foram mais frequentes entre as mães acompanhadas no parto.

A Tabela 4 apresenta a razão de prevalências bruta e ajustada para cada uma das orientações e para a elaboração de um plano de parto, na presença ou não de acompanhante às consultas. Observou-se nos modelos ajustados, exceto nas orientações acerca dos cuidados com o consumo de álcool, tabaco e sinais de risco, que a presença de acompanhante está associada a melhores desfechos. As puérperas que contaram com a presença de acompanhante em suas consultas de pré-natal apresentaram uma prevalência $27 \%$ maior de receber todas as recomendações elencadas pelo Ministério da Saúde, e uma prevalência $51 \%$ maior de elaborar plano de parto com $\mathrm{a}(0)$ profissional que as atendeu.

Ao se analisar o parto e o pós-parto, a razão de prevalências bruta e ajustada demonstrou melhores desfechos para puérperas com acompanhante (Tabela 5). As acompanhadas apresentaram prevalência $11 \%$ maior de amamentação na primeira hora. Observou-se, entretanto, que as puérperas sem acompanhante reportaram prevalência 2,13 vezes maior de serem amarradas durante o parto. Entre as puérperas que realizaram parto vaginal, as prevalências de receber manobra não farmacológica, receber analgesia e poder escolher a posição para o parto foram, respectivamente, $96 \%, 189 \%$ e $63 \%$ maiores entre as puérperas que estavam com acompanhante.

\section{Discussão}

Seis em cada dez puérperas contaram com a presença de acompanhante durante suas consultas de pré-natal; e nove em cada dez, durante 0 parto. Puérperas acompanhadas apresentaram maior probabilidade de receber dos profissionais de saúde todas 
Tabela 1 - Distribuição da amostra de puérperas que realizaram o pré-natal e parto pelo Sistema Único de Saúde $(n=3.580)$, Santa Catarina, 2019

\begin{tabular}{|c|c|}
\hline Variável & n (\%) \\
\hline \multicolumn{2}{|c|}{ Faixa etária (anos) ( $n=3.524)$} \\
\hline 13-19 & $472(13,4)$ \\
\hline 20-35 & $2.653(75,3)$ \\
\hline $36-46$ & $399(11,3)$ \\
\hline \multicolumn{2}{|c|}{ Mora com companheiro $(n=3.559)$} \\
\hline Sim & $2.864(80,5)$ \\
\hline Não & $695(19,5)$ \\
\hline \multicolumn{2}{|c|}{ Gravidez planejada ( $\mathrm{n}=3.551$ ) } \\
\hline Sim & $1.421(40,0)$ \\
\hline Não & $2.130(60,0)$ \\
\hline \multicolumn{2}{|c|}{ Raça/cor da pele ( $n=3.476$ ) } \\
\hline Branca & $2.205(63,4)$ \\
\hline Preta & $330(9,5)$ \\
\hline Parda & $941(27,1)$ \\
\hline \multicolumn{2}{|c|}{ Escolaridade (anos de estudo) ( $n=3.476$ ) } \\
\hline$\geq 13$ & $458(13,0)$ \\
\hline $10-12$ & $1.853(52,5)$ \\
\hline$\leq 9$ & $1,218(34,5)$ \\
\hline \multicolumn{2}{|c|}{ Renda per capita $(\mathrm{n}=3.395)$} \\
\hline Tercil mais rico & $1.114(32,8)$ \\
\hline Tercil intermediário & $1.147(33,8)$ \\
\hline Tercil mais pobre & $1.134(33,4)$ \\
\hline \multicolumn{2}{|c|}{ Número de consultas de pré-natal $(n=3.428)$} \\
\hline $1-3$ & $88(2,6)$ \\
\hline 4-6 & $606(17,7)$ \\
\hline 7 ou mais & $2.734(79,7)$ \\
\hline \multicolumn{2}{|c|}{ Tipo de parto $(n=3.574)$} \\
\hline Vaginal & $2.044(57,2)$ \\
\hline Cesárea & $1.530(42,8)$ \\
\hline \multicolumn{2}{|c|}{ Estava acompanhada durante as consultas de pré-natal ( $n=3.514)$} \\
\hline Sim & $2.143(61,0)$ \\
\hline Não & $1.371(39,0)$ \\
\hline \multicolumn{2}{|c|}{ Estava acompanhada durante o parto e no pós-parto ( $n=3.573$ ) } \\
\hline Sim & $3.254(91,1)$ \\
\hline Não & $319(8,9)$ \\
\hline
\end{tabular}


Tabela 2 - Distribuição da amostra de mulheres que realizaram o pré-natal e parto pelo Sistema Único de Saúde (n=3.580) e percentual de puérperas que receberam orientações e elaboraram plano de parto, segundo a presença ou não de acompanhante no pré-natal, Santa Catarina, 2019

\begin{tabular}{|c|c|c|c|c|c|c|}
\hline \multirow{3}{*}{$\begin{array}{l}\text { Orientações durante o pré-natal } \\
\text { e elaboração de plano de parto }\end{array}$} & \multirow{2}{*}{\multicolumn{2}{|c|}{ Total }} & \multicolumn{4}{|c|}{ Presença de acompanhante no pré-natal } \\
\hline & & & \multicolumn{2}{|r|}{ Sim } & \multicolumn{2}{|r|}{ Não } \\
\hline & $\mathbf{n}$ & $\%\left(I C_{95 \%}{ }^{a}\right)$ & $\mathbf{n}$ & $\%\left(\mathrm{IC}_{95 \%}{ }^{\mathrm{a}}\right)$ & $\mathbf{n}$ & $\%\left(I C_{95 \%}{ }^{a}\right)$ \\
\hline \multicolumn{7}{|l|}{ A gestante foi orientada sobre } \\
\hline Importância da amamentação exclusiva até os 6 meses & 2.181 & $61,9(60,3 ; 63,5)$ & 1.361 & $64,1(62,0 ; 66,1)$ & 792 & $58,4(55,8 ; 61,0)$ \\
\hline Como posicionar 0 bebê para mamar & 1.636 & $46,6(44,9 ; 48,2)$ & 1.021 & $48,3(46,2 ; 50,4)$ & 591 & $43,7(41,0 ; 46,3)$ \\
\hline Importância da realização de atividade física & 2.109 & $60,1(58,4 ; 61,7)$ & 1.324 & $62,6(60,5 ; 64,7)$ & 756 & $55,9(53,2 ; 58,5)$ \\
\hline Riscos de utilizar medicamentos durante a gravidez & 2.722 & $77,2(75,7 ; 78,5)$ & 1.676 & $78,9(77,1 ; 80,6)$ & 1.010 & $74,3(71,9 ; 76,6)$ \\
\hline Riscos do consumo de álcool durante a gravidez & 2.642 & $75,0(73,5 ; 76,4)$ & 1.065 & $75,7(73,8 ; 77,5)$ & 1.001 & $73,7(71,3 ; 76,0)$ \\
\hline Riscos do consumo de tabaco durante a gravidez & 2.663 & $75,6(74,1 ; 77,0)$ & 1.624 & $76,6(74,8 ; 78,4)$ & 1.002 & $73,7(71,3 ; 76,0)$ \\
\hline $\begin{array}{l}\text { Os sinais de risco na gravidez para os quais se deve procurar } \\
\text { por assistência }\end{array}$ & 2.831 & $80,5(79,1 ; 81,7)$ & 1.731 & $81,6(80,0 ; 83,2)$ & 1.066 & $78,7(76,5 ; 80,8)$ \\
\hline Sinais de início do trabalho de parto & 2.255 & $64,0(62,4,65,6)$ & 1.408 & $66,4(64,4 ; 68,4)$ & 816 & $60,2(57,5 ; 62,8)$ \\
\hline $\begin{array}{l}0 \text { que se pode fazer durante } 0 \text { trabalho de parto para facilitar } \\
\text { o nascimento }\end{array}$ & 1.828 & $52,0(50,3 ; 53,6)$ & 1.144 & $54,1(52,0 ; 56,2)$ & 654 & $48,2(45,6 ; 50,9)$ \\
\hline $\begin{array}{l}\text { A possibilidade de se ter um acompanhante no momento } \\
\text { do parto }\end{array}$ & 2.312 & $65,5(63,9 ; 67,0)$ & 1.446 & $68,0(66,0 ; 70,0)$ & 832 & $61,2(58,6 ; 63,8)$ \\
\hline A possibilidade de visitar a maternidade durante o pré-natal & 1.421 & $40,3(38,7 ; 41,9)$ & 899 & $42,3(40,3 ; 44,5)$ & 499 & $36,7(34,2 ; 39,3)$ \\
\hline Recebeu todas as recomendações supracitadas & 616 & $17,5(16,3 ; 18,8)$ & 398 & $18,8(17,2 ; 20,6)$ & 207 & $15,2(13,4 ; 17,2)$ \\
\hline $\begin{array}{l}\text { Foi elaborado plano de parto com a(o) } \\
\text { profissional de saúde }\end{array}$ & 261 & $\begin{array}{c}7,4 \\
(6,6 ; 8,3)\end{array}$ & 179 & $\begin{array}{c}8,5 \\
(7,4 ; 9,8)\end{array}$ & 78 & $\begin{array}{c}5,8 \\
(4,7 ; 7,2)\end{array}$ \\
\hline
\end{tabular}

a) $I_{95 \%}$ : intervalo de confiança de $95 \%$.

as orientações para o pré-natal aqui analisadas, e de construir, junto a essas e esses profissionais, um plano de parto. Estar acompanhada no parto também se associou a maiores prevalências de boas práticas assistenciais, como amamentação na primeira hora de vida, escolha da posição de expulsão, não ser amarrada, submissão a manobra não farmacológica e analgesia para alívio da dor.

0 presente trabalho de pesquisa corrobora diferentes estudos que apontaram a importância da presença de acompanhante desde atenção pré-natal, com seus efeitos positivos nas atitudes, sentimentos e percepção da gestação pela mulher, proporcionando melhor experiência de parto e puerpério. Aquelas que podem contar com a presença de acompanhante durante 0 pré-natal são, igualmente, as que terão a maior possibilidade de contar com acompanhante durante 0 período do trabalho de parto e parto. ${ }^{6,13,15}$
Contudo, a presença de acompanhante no cenário do pré-natal sofre limitações, possivelmente ocasionadas por fatores familiares maternos e/ou institucionais. Isto ocorre, principalmente, porque essa prática ainda não se consolidou em todas as instituições e mentes dos indivíduos e, portanto, deve ser revista no sentido de o acompanhante ser de livre escolha da gestante, não vinculada a outros condicionantes eventualmente presentes. ${ }^{3}$

A assistência de acompanhante durante o processo de parto tem contribuído para atenuar as desigualdades sociais no atendimento e melhorar a percepção das mulheres sobre o cuidado recebido., ${ }^{2,15}$ A gestante, geralmente, chega à maternidade acompanhada no momento da internação; porém, um afastamento gradual é possível de se observar, de acordo com a evolução das etapas do processo de parturição, o que pode ter relação com a não inclusão de acompanhante 
Tabela 3 - Distribuição da amostra de puérperas que realizaram o pré-natal e parto pelo Sistema Único de Saúde ( $n=3.580)$ e prevalência de práticas obstétricas realizadas durante o parto, segundo a presença ou não de acompanhante, Santa Catarina, 2019

\begin{tabular}{|c|c|c|c|c|c|c|}
\hline \multirow{3}{*}{ Práticas obstétricas durante o parto } & \multirow{2}{*}{\multicolumn{2}{|c|}{ TOTAL }} & \multicolumn{4}{|c|}{ Presença de acompanhante no pré-natal } \\
\hline & & & \multicolumn{2}{|c|}{ Sim } & \multicolumn{2}{|c|}{ Não } \\
\hline & $\mathbf{n}$ & $\%\left({ }^{\prime C} C_{95 \%}{ }^{a}\right)$ & $\mathbf{n}$ & $\%\left(\right.$ IC $\left._{95 \%}{ }^{a}\right)$ & $\mathbf{n}$ & $\%\left(I_{95 \%}{ }^{a}\right)$ \\
\hline Amamentou na primeira hora & 2.394 & $71,2(69,7 ; 72,7)$ & 2.206 & $71,8(70,2 ; 73,4)$ & 187 & $64,9(59,2 ; 70,2)$ \\
\hline Foi amarrada durante o parto & 300 & $9,2(8,2 ; 10,2)$ & 248 & $8,3(7,4 ; 9,4)$ & 52 & $18,5(14,4 ; 23,5)$ \\
\hline Foi realizada episiotomiab & 286 & $14,3(12,8 ; 15,9)$ & 267 & $14,3(12,8 ; 16,0)$ & 19 & $14,5(9,4 ; 21,7)$ \\
\hline Pôde escolher a posiçãob & 1.017 & $49,0(46,8 ; 51,2)$ & 929 & $50,1(47,8 ; 52,4)$ & 88 & $32,3(24,8 ; 40,9)$ \\
\hline Recebeu MNF' para alívio da dor ${ }^{b}$ & 1.066 & $52,7(50,5 ; 54,9)$ & 1.033 & $54,7(52,5 ; 57,0)$ & 33 & $24,4(17,9 ; 32,5)$ \\
\hline Chuveiro & 984 & $48,7(46,5 ; 50,9)$ & 957 & $50,8(48,5 ; 53,0)$ & 27 & $20,0(14,0 ; 27,7)$ \\
\hline Bola & 694 & $34,3(32,3 ; 36,4)$ & 678 & $35,9(33,8 ; 38,1)$ & 16 & $11,9(7,4 ; 18,7)$ \\
\hline Massagem & 498 & $24,6(22,8 ; 26,6)$ & 484 & $25,7(23,8 ; 27,7)$ & 14 & $10,4(6,2 ; 16,8)$ \\
\hline Analgesia $^{b}$ & 371 & $18,4(16,8 ; 20,2)$ & 363 & $19,3(17,6 ; 21,2)$ & 8 & $5,9(3,0 ; 11,5)$ \\
\hline
\end{tabular}

a) $\mathrm{IC}_{95 \mathrm{~S}}$ : intervalo de confiança de $95 \%$.

b) Apenas para mulheres que realizaram parto vaginal.

c) MNF: medida não farmacológica.

Tabela 4 - Associação entre recebimento de orientações durante o pré-natal e elaboração de plano de parto com a presença de acompanhante no pré-natal, em amostra de puérperas que realizaram o pré-natal e parto pelo Sistema Único de Saúde ( $\mathrm{n}=3.580)$, Santa Catarina, 2019

\begin{tabular}{|c|c|c|c|c|}
\hline Orientações durante o pré-natal e elaboração de plano de parto & RPa bruta $\left(\mathrm{IC}_{95 \%}{ }^{\mathrm{b}}\right)$ & p-valor & RPa ajustada (IC $\left.{ }_{95 \%}^{b}\right)$ & p-valor \\
\hline \multicolumn{5}{|l|}{ A gestante foi orientada sobre } \\
\hline Importância da amamentação exclusiva até os 6 meses & $1,10(1,04 ; 1,16)$ & 0,001 & $1,12(1,05 ; 1,18)$ & $<0,001$ \\
\hline Como posicionar 0 bebê para mamar & $1,10(1,03 ; 1,19)$ & 0,009 & $1,15(1,06 ; 1,24)$ & 0,001 \\
\hline Importância da realização de atividade física & $1,12(1,06 ; 1,19)$ & $<0,001$ & $1,11(1,04 ; 1,18)$ & 0,001 \\
\hline Riscos de utilizar medicamentos durante a gravidez & $1,06(1,02 ; 1,10)$ & 0,002 & $1,05(1,01 ; 1,10)$ & 0,011 \\
\hline Riscos do consumo de álcool durante a gravidez & $1,03(0,99 ; 1,07)$ & 0,189 & $1,02(0,97 ; 1,06)$ & 0,447 \\
\hline Riscos do consumo de tabaco durante a gravidez & $1,04(1,00 ; 1,08)$ & 0,058 & $1,02(0,98 ; 1,07)$ & 0,264 \\
\hline Os sinais de risco na gravidez que devem fazer procurar por assistência & $1,04(1,00 ; 1,07)$ & 0,037 & $1,03(0,99 ; 1,06)$ & 0,159 \\
\hline Sinais de início do trabalho de parto & $1,10(1,05 ; 1,16)$ & $<0,001$ & $1,10(1,04 ; 1,16)$ & 0,001 \\
\hline $\begin{array}{l}0 \text { que se pode fazer durante } 0 \text { trabalho de parto para facilitar } 0 \\
\text { nascimento }\end{array}$ & $1,12(1,05 ; 1,20)$ & 0,001 & $1,11(1,04 ; 1,20)$ & 0,003 \\
\hline A possibilidade de se ter um acompanhante no momento do parto & $1,11(1,06 ; 1,17)$ & $<0,001$ & $1,11(1,05 ; 1,17)$ & $<0,001$ \\
\hline A possibilidade de visitar a maternidade durante o pré-natal & $1,15(1,06 ; 1,26)$ & 0,001 & $1,14(1,04 ; 1,25)$ & 0,004 \\
\hline Recebeu todas as recomendações supracitadas & $1,24(1,06 ; 1,44)$ & 0,006 & $1,27(1,08 ; 1,50)$ & 0,003 \\
\hline $\begin{array}{l}\text { Foi elaborado plano de parto com a(0) } \\
\text { profissional de saúde }\end{array}$ & $1,47(1,13 ; 1,89)$ & 0,004 & $1,51(1,15 ; 1,97)$ & 0,003 \\
\hline
\end{tabular}

a) RP: razão de prevalências.

b) $\mathrm{IC}_{95 \%}$ : intervalo de confiança de $95 \%$.

c) Teste de Wald.

d) Análises ajustadas por faixa etária, residência com companheiro(a), planejamento da gravidez, raça/cor da pele autorreferida, anos de estudo, renda per capita e número de consultas. 
Tabela 5 - Associação de práticas obstétricas realizadas durante o parto com a presença de acompanhante, em amostra de puérperas que realizaram pré-natal e parto pelo Sistema Único de Saúde (n=3.580), Santa Catarina, 2019

\begin{tabular}{|c|c|c|c|c|}
\hline Práticas obstétricas durante o parto & RPa bruta $\left(\mathrm{IC}_{95 \%}{ }^{\mathrm{b}}\right)$ & p-valor & RPa ajustada $\left(\mathrm{IC}_{95 \%}{ }^{\mathrm{b}}\right)$ & p-valor \\
\hline Amamentou na primeira hora & $1,11(1,01 ; 1,21)$ & 0,024 & $1,11(1,02 ; 1,22)$ & 0,022 \\
\hline Foi amarrada durante o parto & $0,45(0,34 ; 0,49)$ & $<0,001$ & $0,47(0,35 ; 0,63)$ & $<0,001$ \\
\hline Foi realizada episiotomia & $1,00(0,93 ; 1,08)$ & 0,947 & $1,02(0,94 ; 1,10)$ & 0,631 \\
\hline Pôde escolher a posição ${ }^{e}$ & $1,55(1,20 ; 2,00)$ & 0,001 & $1,63(1,24 ; 2,16)$ & 0,001 \\
\hline Recebeu MNF para alívio da dor & $2,24(1,66 ; 3,02)$ & $<0,001$ & $1,96(1,44 ; 2,65)$ & $<0,001$ \\
\hline Chuveiro & $2,54(1,81 ; 3,57)$ & $<0,001$ & $2,22(1,56 ; 3,13)$ & $<0,001$ \\
\hline Bola & $3,01(1,89 ; 4,79)$ & $<0,001$ & $2,63(1,63 ; 4,25)$ & $<0,001$ \\
\hline Massagem & $2,48(1,50 ; 4,09)$ & $<0,001$ & $2,05(1,21 ; 3,46)$ & 0,007 \\
\hline Analgesia ${ }^{e}$ & $3,26(1,65 ; 6,42)$ & 0,001 & $2,89(1,40 ; 5,97)$ & 0,004 \\
\hline \multicolumn{5}{|c|}{$\begin{array}{l}\text { a) RP: razão de prevalências. } \\
\text { b) IC } 955_{6} \text { intervalo de confiança de } 95 \% \text {. } \\
\text { c) Teste de Wald. } \\
\text { d) Análises ajustadas por faixa etária, residência com companheiro(a), planejamento da gravidez, raça/cor da pele autorreferida, anos de estudo, renda per capita e número de consultas. } \\
\text { e) Apenas para mulheres que realizaram parto vaginal. } \\
\text { f) MNF: medida não farmacológica. }\end{array}$} \\
\hline
\end{tabular}

na rotina hospitalar. ${ }^{16}$ Para que a gestante/parturiente tenha acesso ao apoio contínuo de acompanhante, este(a) precisa ser instruído(a), incluído em atividades de educação em saúde realizadas em grupos e nas consultas de pré-natal. ${ }^{17}$ Apesar disso, estudos nacionais, sobre dados da pesquisa Nascer no Brasil (2011/2012), demostraram que $24,5 \%$ das mulheres não tiveram acompanhante durante 0 trabalho de parto - valor 2,7 vezes superior à prevalência encontrada neste estudo - e apenas 19,3\% contaram com a presença de acompanhante em todos os momentos da internação. ${ }^{2,18}$ Tais resultados demonstram a heterogeneidade das práticas assistenciais no país. Com base nos últimos dados nacionais, e reconhecidos avanços como a progressão - ainda que lenta - da presença de acompanhantes, uma parcela das mulheres atendidas não conta com esse apoio durante o trabalho de parto e parto, não obstante tal presença ser garantida em lei.

$\mathrm{Na}$ análise sobre 0 recebimento de orientações no acompanhamento pré-natal, verificou-se baixa frequência da orientação da(o) profissional de saúde sobre a possibilidade de a gestante visitar a maternidade onde ocorrerá 0 parto. Estudo nacional sobre a atenção pré-natal e ao parto, a partir de dados da mesma pesquisa Nascer no Brasil de 2011/2012, reforça a importância dessa e de outras orientações: mulheres informadas durante 0 pré-natal e que realizaram a vinculação à maternidade durante 0 acompanhamento podem levar vantagem na presença de acompanhante durante toda a internação para 0 parto. ${ }^{19} 0$ destaque para essas orientações surge a partir da implantação da Rede Cegonha, com o intuito de vincular a gestante à unidade de maternidade $\mathrm{e}$ evitar peregrinação, prevenindo dificuldades de acesso e agilizando a assistência. 0(a) acompanhante, durante esse processo, também deve receber informações dos dispositivos legais e orientações para essa vinculação, podendo contribuir positivamente no acesso a esses serviços.

A orientação sobre amamentação exclusiva até os 6 meses de idade foi oferecida a mais da metade das puérperas do estudo, e ter acompanhante no pré-natal esteve associado a maior chance de receber essa orientação. Tal achado demonstra baixa prevalência quando comparada à dos $91,0 \%$ reportados para essa orientação no Brasil, segundo um estudo nacional cujo objetivo foi avaliar a qualidade da atenção pré-natal no país com dados do Programa Nacional de Melhoria do Acesso e da Qualidade da Atenção Básica (PMAQ) (2012/2013). Quanto à orientação sobre 0 posicionamento do bebê para amamentação, ela foi relatada por apenas $46,6 \%$ das mulheres consultadas. ${ }^{20}$ 0s tópicos que versam sobre amamentação deveriam ser mais presentes na assistência ao pré-natal, tendo 
em vista a importância da amamentação como forma de fortalecimento do vínculo entre a mãe e $o$ bebê, do crescimento e desenvolvimento da criança. ${ }^{20}$

0 (a) acompanhante que participa das consultas de pré-natal pode oferecer maior apoio à mulher durante 0 processo de amamentação, podendo contribuir para sua satisfação com o evento e manutenção do aleitamento. Conforme indicou estudo realizado no Distrito Federal, mulheres com acompanhante no momento do parto apresentaram prevalência de amamentação na primeira hora de vida superior a 70,0\%, sendo $11,0 \%$ maior que a mesma prevalência entre aquelas que não estavam acompanhadas durante 0 parto. ${ }^{21}$

Maior magnitude de razão de prevalências foi observada no desfecho relativo à elaboração do plano de parto. Esta prática foi mais comum nas puérperas que contaram com a presença de acompanhante durante o pré-natal. 0 plano de parto é construído com 0 auxílio $\mathrm{da}(0)$ profissional que realiza o pré-natal, configurando um instrumento educativo, de caráter legal, desenvolvido durante o período gestacional, de acordo com a condição clínica da mulher e a realidade do serviço, com o objetivo de promover a reflexão e auxiliar a gestante na tomada de decisões sobre o parto e os procedimentos a realizar. ${ }^{22,23}$ Recomendado pela OMS, e pelo Ministério da Saúde após a implantação da Rede Cegonha, o plano de parto favorece o empoderamento feminino, promove maior satisfação com o parto e melhores resultados maternos/neonatais; a adesão do instrumento aos serviços de saúde e sua construção durante o pré-natal contribui para a qualidade do cuidado materno-infantil prestado. ${ }^{23}$

0 presente estudo demonstrou que a presença de acompanhante no momento do trabalho de parto foi associada à realização de manobras não farmacológicas. 0 mesmo resultado foi apontado por estudo realizado em três maternidades públicas do município de Niterói, RJ, onde se observou maior ocorrência de massagens, banhos, técnicas de respiração e movimentação nas parturientes que contaram com acompanhantes de sua livre escolha. ${ }^{16}$

As boas práticas de atenção ao parto e nascimento orientam sobre o que deve ou não ser feito no processo do parto. Práticas identificadas como violência obstétrica são desestimuladas. Esse tipo de violência pode se expressar de forma verbal, física, no uso inadequado de tecnologias, intervenções e procedimentos desnecessários, com a possibilidade, inclusive, de desencadear sequelas físicas e psicológicas. ${ }^{24}$ Ter sido amarrada durante 0 parto foi ato relatado por aproximadamente uma em cada dez puérperas entrevistadas, sendo menos prevalente entre mulheres com acompanhante, o que demonstra a importância de a mulher ser acompanhada por alguém de sua livre escolha nesse momento tão delicado, para sua maior segurança, proteção e auxílio na tomada de decisão. Ainda que, por vezes, os atos de amarrar braços ou pernas sejam justificados com o propósito de impedir contaminação do campo cirúrgico, eles são desnecessários e configuram violência obstétrica. Eles também não devem ser praticados porque, entre outros fatores, violam a autonomia e bem-estar da mulher e comprometem sua possibilidade de acolher nos braços a criança recém-nascida. ${ }^{25}$

Do total de puérperas que apresentaram parto vaginal, aproximadamente metade pôde escolher a posição para expulsão, enquanto aquelas que não estavam com acompanhante exerceram essa autonomia em cerca de um terço dos partos. Estudo realizado na cidade de São Paulo não identificou como prática entre os profissionais de saúde oferecer à parturiente a possibilidade de utilizar posições variadas no momento da expulsão, costumando adotar a posição de litotomia (posição ginecológica, deitada com as pernas elevadas em apoio) como padrão, por facilitar 0 acesso ao canal de parto e a realização de intervenções; e por presumirem ser essa a posição de preferência das mulheres. ${ }^{26}$ Estudo qualitativo, realizado em um hospital universitário do estado de Santa Catarina, analisou a percepção da puérpera sobre a experiência de parir em posição vertical ou horizontal, e concluiu: a posição vertical foi considerada pela mulher como a mais positiva, por favorecer a movimentação, ser mais adequada para realização da força, descida e expulsão do feto, além de permitir sua maior participação no parto. ${ }^{27}$ Neste contexto, observa-se que os conhecimentos acerca do parto devem ter início no pré-natal e, uma vez reforçados durante toda a gestação até 0 parto, podem oferecer à gestante e acompanhante 0 empoderamento e a autonomia convenientes à parturição, influenciando positivamente na maneira como ambos vivenciam a consecução da gestação.

Destacam-se como limitações do estudo o fato de as entrevistas terem se realizado dentro das 48 horas após o parto, durante a internação da puérpera. Esta condição para a coleta dos dados pode gerar um viés 
de cortesia e, assim, influenciar as respostas sobre as práticas de atenção ao parto; outra possível limitação está no fato de as informações sobre as orientações serem autorreferidas, sujeitas a viés de memória. Ademais, a informação sobre dispor de acompanhante diz respeito a essa presença em geral, sem especificar entre pré-parto, parto e pós-parto, o que pode superestimar os resultados da pesquisa.

A presença de acompanhante no parto já é reconhecida como uma boa prática obstétrica, um direito garantido pela legislação, que, entretanto, não contempla a presença de acompanhante nas consultas de pré-natal. A despeito das limitações consideradas, o estudo concluiu que a adoção dessa prática pode contribuir para a qualificação da assistência durante a gestação; e mais além, na amamentação até a primeira hora de vida do bebê. Contudo, há pouca mobilização da sociedade - empresas, governos e serviços de saúde - para estimular e viabilizar a presença de acompanhante também nas consultas de pré-natal. É preciso que esse ponto seja considerado, de maneira mais forte e consistente, e adotado na agenda de cuidados a serem prestados à gestante e à criança no país.
Por fim, observou-se que a presença de acompanhante junto à mulher nas consultas de pré-natal e no parto está associada à maior qualidade da assistência recebida pelas usuárias do Sistema Único de Saúde no estado de Santa Catarina. Os achados deste estudo contribuem para uma reflexão mais ampla sobre a prática assistencial e a importância da disposição dos serviços de saúde para incentivar e facilitar a participação de acompanhantes junto à mulher, e avaliar, em suas rotinas, as razões para mulheres acompanhadas apresentarem maior probabilidade de receber melhores cuidados assistenciais.

\section{Contribuição dos autores}

Tomasi YT, Saraiva SS, Boing AF e Boing AC contribuíram na concepção e delineamento do estudo, análise e interpretação dos dados e redação da primeira versão do manuscrito. Delziovo CR e Wagner KJP contribuíram na análise e interpretação dos dados e revisão crítica do manuscrito. Todos os autores aprovaram a versão final do manuscrito e são responsáveis por todos os aspectos do trabalho, incluindo a garantia de sua precisão e integridade.

\section{Referências}

1. Hodnett ED, Gates S, Hofmeyr GJ, Sakala C. Continuous support for women during childbirth. Cochrane Database Syst Rev [Internet]. 2013 Jul [cited 2020 Nov 6]; 7(7):CD003766. Available from: https://doi.org/10.1002/14651858.cd003766.pub6

2. Diniz CSG, d'Orsi E, Domingues RMSM, Torres JA, Dias MAB, Schneck CA, et al. Implementação da presença de acompanhantes durante a internação para o parto: dados da pesquisa nacional Nascer no Brasil. Cad Saúde Pública [Internet]. 2014 [citado 2020 nov 6]; 30(suppl 1):S140-53. Disponível em: http://dx.doi.org/10.1590/0102-311X00127013

3. Organização Mundial da Saúde - OMS. Boas práticas de atenção ao parto e ao nascimento [Internet]. [S.1.]: 0MS; 1996 [citado 2020 nov 6]. 3 p. Disponível em: http://static.hmv.org.br/wp-content/ uploads/2014/07/0MS-Parto-Normal.pdf

4. Brasil. Presidência da República. Lei no 11.108, de 7 de abril de 2005. Garante as parturientes 0 direito à presença de acompanhante durante 0 trabalho de parto, parto e pós-parto imediato, no

âmbito do SUS [Internet]. Diário Oficial da União, Brasília (DF), 2005 abr 8 [citado 2020 nov 6]. Disponível em: http://www.planalto.gov.br/ccivil_03/_Ato2004-2006/2005/Lei/L11108.htm

5. Brasil. Ministério da Saúde. Portaria no 1.459, de 24 de junho de 2011. Institui a Rede Cegonha [Internet]. Diário Oficial da União, Brasília (DF), 2011 jun 25 [citado 2020 nov 6]. Disponível em: http://bvsms.saude.gov.br/bvs/saudelegis/ gm/2011/prt1459_24_06_2011.html

6. Holanda SM, Castro RCMB, Aquin PS, Pinheiro AKB, Lopes LG, Martins ES. Influência da participação do companheiro no pré-natal: satisfação de primíparas quanto ao apoio no parto. Texto-Contexto Enferm [Internet]. 2018 [citado 2020 nov 6];27(2):1-10. Disponível em: https:// doi.org/10.1590/0104-070720180003800016

7. Mallmann MB, Boing AF, Tomasi YT, Anjos JC, Boing AC. Evolução das desigualdades socioeconômicas na realização de consultas de pré-natal entre parturientes brasileiras: análise do período 2000-2015. 
Epidemiol Serv Saúde [Internet]. 2018 [citado 2020 nov 6];27(4):e2018022. Disponível em: http:// dx.doi.org/10.5123/s1679-49742018000400014

8. Araújo ICFG, Ferreira TLS, Araújo DV, Melo KDF, Andrade FB. Qualidade do parto e impacto nos indicadores da saúde da criança. Rev Ciênc Plur [Internet]. 2019 jun [citado 2020 nov 6];5(1):18-33. Disponível em: https://doi.org/10.21680/2446-7286.2019v5n1ID17943

9. Monguilhott JJC, Brüggemann OM, Freitas PF, d'Orsi E. Nascer no Brasil: a presença do acompanhante favorece a aplicação das boas práticas na atenção ao parto na região Sul. Rev Saúde Pública [Internet]. 2018 [citado 2020 nov 6];52:1. Disponível em: https:// doi.org/10.11606/s1518-8787.2018052006258

10. Líbera BD, Saunders C, Santos MMAS, Rimes KA, Brito FRSS, Baião MR. Avaliação da assistência pré-natal na perspectiva de puérperas e profissionais de saúde. Ciênc Saúde Coletiva [Internet]. 2011 [citado 2020 nov 6];16(12):4855-64. Disponível em: https:// doi.org/10.1590/S1413-81232011001300034

11. Brüggemanna OM, Ebeleb RR, Ebsenc ES, Batistab BD. No parto vaginal e na cesariana acompanhante não entra: discursos de enfermeiras e diretores técnicos. Rev Gaúcha Enferm [Internet]. 2015 [citado 2020 nov 6];36(esp):152-58. Disponível em: http:// dx.doi.org/10.1590/1983-1447.2015.esp.53019

12. Poh HL, Koh SSL, Seow HCL, He HG. First-time fathers' experiences and needs during pregnancy and childbirth: a descriptive qualitative study. Midwifery [Internet] 2014 Jun [cited 2020 Nov 6];30(6):779-87. Available from: https:// doi.org/10.1016/j.midw.2013.10.002

13. Perdomini FRI, Bonilha ALL. A participação do pai como acompanhante da mulher no parto. Texto-Contexto Enferm [Internet]. 2011 [citado 2020 nov 6];20(3):445-52. Disponível em: https:// doi.org/10.1590/S0104-07072011000300004

14. Ministério da Saúde (BR). Secretaria de Atenção à Saúde. Departamento de Atenção Básica. Atenção ao pré-natal de baixo risco [Internet]. Brasília: Ministério da Saúde; 2012 [citado 2020 nov 6]. Disponível em: http://bvsms.saude.gov.br/bvs/publicacoes/ cadernos_atencao_basica_32_prenatal.pdf

15. Santana MA, Souza SRRK, Gualda DMR, Wall ML. Perfil de gestantes e acompanhantes das oficinas para o parto acompanhado. Cogitare Enferm [Internet]. 2012 mar [citado 2020 nov 6];17(1):106-12. Disponível em: https://doi.org/10.5380/ce.v17i1.26382
16. Dulfe PAM, De Lima DVM, Alves VH, Rodrigues DP, Barcellos JG, Cherem EDO. Presença do acompanhante de livre escolha no processo parturitivo: repercussões na assistência obstétrica. Cogitare Enferm [Internet]. 2016 Jan [citado 2020 nov 6];21(4):1-8. Disponível em: https://doi.org/10.5380/ce.v21i4.37651

17. Gallo RBS, Santana LS, Marcolin AC, Ferreira CHJ, Duarte G, Quintana SM. Recursos não-farmacológicos no trabalho de parto: protocolo assistencial. Femina [Internet]. 2011 jan [citado 2020 nov 6];39(1):41-8. Disponível em: http://files.bvs.br/ upload/S/0100-7254/2011/v39n1/a2404.pdf

18. D’Orsi E, Brüggemann OM, Diniz CSG, Aguiar JM, Gusman CR, Torres JA, et al. Desigualdades sociais e satisfação das mulheres com 0 atendimento ao parto no Brasil: estudo nacional de base hospitalar. Cad Saúde Pública [Internet]. 2014 [citado 2020 nov 6];30(Suppl 1). Disponível em: https://doi.org/10.1590/0102- 311X00087813

19. Leal MC, Gama SGN, Pereira APE, Pacheco VE, Carmo CN, Santos RV. A cor da dor: iniquidades raciais na atenção pré-natal e ao parto no Brasil. Cad Saúde Pública [Internet]. 2017 [citado 2020 nov 6];33 Sup 1:e00078816. Disponível em: https://doi.org/10.1590/0102-311X00078816

20. Tomasi E, Fernandes PAA, Fischer T, Siqueira FCV, Silveira DS, Thumé E, et al. Qualidade da atenção pré-natal na rede básica de saúde do Brasil: indicadores e desigualdades sociais. Cad Saúde Pública [Internet]. 2017 [citado 2020 nov 6];33(3):1-11. Disponível em: http://dx.doi.org/10.1590/0102-311x00195815

21. Bandeira de Sá NN, Gubert MB, Santos W, Santos LMP. Fatores ligados aos serviços de saúde determinam 0 aleitamento materno na primeira hora de vida no Distrito Federal, Brasil, 2011. Rev Bras Epidemiol [Internet]. 2016 set [citado 2020 nov 6];19(3):509-24. Disponível em: https:// doi.org/10.1590/1980-5497201600030004

22. Medeiros RMK, Figueiredo G, Correa ÁCP, Barbieri M. Repercussões da utilização do plano de parto no processo de parturição. Rev Gaúcha Enferm [Internet]. 2019 [citado 2020 nov 6];40:e20180233. Disponível em: https://doi.org/10.1590/1983-1447.2019.20180233

23. Silva WNS, Azevedo JAF, Holanda VR, Gomes ALV, Albuquerque GPM. Plano de parto como instrumento das boas práticas no parto e nascimento: revisão integrativa. Rev Baiana Enferm [Internet]. 2019 [citado 2020 nov 6];33:e32894. Disponível em: https://doi.org/10.18471/rbe.v33.32894 
24. Sena LM, Tesser CD. Violência obstétrica no Brasil e 0 ciberativismo de mulheres mães: relato de duas experiências. Interface - Comun Saúde Educ [Internet]. 2016 nov [citado 2020 nov 6];21(60):209-20. Disponível em: https://doi.org/10.1590/1807-57622015.0896

25. Salgado HO, Niy DY, Diniz CSG. Groggy and with tied hands: the first contact with the newborn according to women that had an unwanted c-section. J Hum Growth Dev [Internet]. 2013 Aug [cited 2020 Nov 6];23(2):190. Available from: https://doi.org/10.7322/jhgd.61298
26. Niy DY, Oliveira VC, Oliveira LR, Alonso BD, Diniz CSG. Como superar a cultura da imobilização física das parturientes? Resultados parciais de estudo de intervenção em São Paulo, SP, Brasil. Interface - Comun Saúde Educ [Internet]. 2019 [citado 2020 nov 6];23:1-16. Disponível em: https://doi.org/10.1590/interface.180074

27. Gayeski ME, Brüggemann OM. Puerperal women's perceptions on vertical and horizontal deliveries. Rev Lat Am Enfermagem [Internet]. 2009 Apr [cited 2020 Nov 6];17(2):153-9. Available from: https:// doi.org/10.1590/S0104-11692009000200003

\section{Abstract}

objective: To analyze association between presence of a companion during prenatal consultations and childbirth and quality of care received by puerperal women using the Brazilian National Health System (SUS). Methods: This was a cross-sectional study with puerperal women who underwent prenatal care and delivery on the SUS in Santa Catarina State, Brazil, in 2019, and who were interviewed within 48 bours postpartum. Prevalence ratios were estimated using Poisson regression. Results: 3,580 puerperal women were interviewed. In prenatal care, presence of a companion was positively associated with receiving guidance from bealth professionals ( $P R=1.27-95 \% C I$ 1.08;1.50) and building a birth plan ( $P R=1.51-95 \% C I$ 1.15;1.97). At delivery, presence of a companion was associated with greater receipt of analgesics $(P R=2.89$ - 95\%CI 1.40;5.97), non-pharmacological pain relief management $(P R=1.96$ - 95\%CI 1.44;2.65), choice of position for delivery (PR=1.63 - 95\%CI 1.24;2.16) and less likelihood of being strapped down ( $P R=0.47-95 \% C I$ 0.35;0.63). Conclusion: Presence of a companion during prenatal care and delivery was associated with better quality of care.

Keywords: Prenatal Care; Humanized Delivery; Brazilian National Health System; Patient Rights; Women's Health; Cross-Sectional Studies.

\section{Resumen}

objetivo: Analizar la asociación entre presencia de acompañante durante atención prenatal y parto con la calidad de atención que reciben las usuarias del Sistema Único de Salud (SUS). Métodos: Estudio transversal con puérperas que recibieron atención prenatal y parto por el SUS en Santa Catarina, Brasil, en 2019, entrevistadas basta 48 boras posparto. Las razones de prevalencia se estimaron mediante la regresión de Poisson. Resultados: Se entrevistaron 3,580 puérperas. En la atención prenatal, la presencia de acompañante se asoció positivamente a recibir orientación de los profesionales de salud $\left(R P=1,27-I C_{95 \%} 1,08 ; 1,50\right)$ y la construcción del plan de parto $\left(R P=1,51-I C_{95 \%} 1,15 ; 1,97\right)$. En el momento del parto, se asoció con mayor recepción de analgesia ( $\left.R P=2,89-I C_{95 \%} 1,40 ; 5,97\right)$, maniobras no-farmacológicas para alivio del dolor $(R P=1,96-$ $I C_{95 \%}$ 1,44;2,65), elección de la posición para el parto $\left(R P=1,63-I C_{95 \%} 1,24 ; 2,16\right)$ y menor probabilidad de estar atada $\left(R P=0,47-I C_{95 \%} 0,35 ; 0,63\right)$. Conclusión: La presencia de acompañante en la atención prenatal y el parto se asoció con una mejor calidad de la atención.

Palabras clave: Atención Prenatal; Parto Humanizado; Sistema Único de Salud; Derechos del Paciente; Salud de la Mujer; Estudios Transversales.

Recebido em 08/06/2020

Aprovado em 06/10/2020 